\title{
Adjetivos e información: la intervención de la subjetividad en la prensa escrita digital de América Latina
}

\author{
Silvia Ramírez Gelbes \\ Universidad de San Andrés, Argentina \\ sgelbes@udesa.edu.ar \\ Patricio Dillon \\ Universidad de San Andrés, Argentina \\ pdillon@udesa.edu.ar \\ Rodrigo Gil Buetto \\ Universidad de San Andrés, Argentina \\ rodrigb_96@hotmail.com

\section{Mattia Panza} \\ Universidad de San Andrés, Argentina \\ panzamattia@gmail.com
}

\author{
Tomás Bombau \\ Universidad de San Andrés, Argentina \\ tomas.bombau@gmail.com \\ Bianca Di Virgilio \\ Universidad de San Andrés, Argentina \\ bdivirgilio@udesa.edu.ar \\ Pilar Llambías \\ Universidad de San Andrés, Argentina \\ llambias.pilar@gmail.com \\ Victoria Siano \\ Universidad de San Andrés, Argentina \\ vsiano@udesa.edu.ar
}

Recibido: 5/3/2018 / Aceptado:11/4/2018

doi: 10.26439/contratexto2018.n029.1856

Resumen. La prescripción ha aludido tradicionalmente a la noción de periodista como la figura capaz de reproducir en su discurso la realidad tal y como es, de manera objetiva, por medio de enunciados que la describen fielmente. Pero la subjetividad no está en absoluto ausente en los textos efectivamente producidos por los periodistas de algunos países de América Latina. Si tomamos, por ejemplo, el empleo de los adjetivos calificativos - una de las vías privilegiadas para la inscripción de la subjetividad-, algunos estudios (Amado et al., 2016) enseñan que la prensa argentina y la chilena incluyen en sus notas informativas más adjetivos calificativos que cualquier otra prensa de la región, frente a la de Ecuador, que escasamente los introduce en sus notas. Luego de caracterizar y clasificar los adjetivos calificativos, y con el fin de confirmar -o refutaresa descripción, estudiamos 490 notas informativas escritas aparecidas en tres diarios online argentinos y en cuatro medios diferentes de sendos países latinoamericanos. Nuestros resultados, que confirman parcialmente los hallazgos de esos estudios previos, son interpretados a la luz de una visión del periodismo como orientado a cumplir con una misión social, e investido de un compromiso individual con su audiencia.

Palabras clave: subjetividad / adjetivos / periodismo / periodismo digital / nota informativa 


\title{
Adjectives and Information: Subjectivity Intervention in Latin American Digital Written Press
}

\begin{abstract}
Prescription has traditionally referred to the idea of the journalist as the figure capable of reproducing reality in his or her discourse just as it is, objectively, by means of statements that describe it faithfully. But subjectivity is not absent in the texts effectively produced by journalists in some Latin American countries. If we take as an example the use of qualifying adjectives - one of the privileged ways to register subjectivity-, some studies (Amado et al., 2016) show that the Argentine and Chilean press include, in their press releases, more qualifying adjectives than any other press in the region, such as that of Ecuador, which scarcely introduces them in their press releases. After characterizing and classifying the qualifying adjectives, and in order to confirm -or reject- that description, we studied 490 written press releases of three Argentine online newspapers and four different online media of Latin American countries. Our results, which partially confirm the findings of those previous studies, are interpreted by considering journalism as oriented to fulfill a social mission and invested with an individual commitment towards its audience.
\end{abstract}

Keywords: subjectivity / adjectives / journalism / digital journalism / press release 


\section{Introducción}

$\mathrm{E}$ ntre otras condiciones, la competencia pragmática de los autores profesionales se manifiesta en la habilidad de emplear los recursos retóricos propios de su campo de trabajo, en la medida en que, de ese modo, se alinean en lo discursivo con las propiedades identitarias de los roles construidos socialmente dentro de su comunidad. Desde esa perspectiva, no puede desdeñarse el papel que cumple la prescripción en el campo específico. Así, por ejemplo, se enseña que el empleo de formas personales debe ser evitado en la escritura académica (Hyland, 2002, p. 1095) o se "decreta" la orientación hacia un discurso objetivo -despojado de marcas de subjetividad- en la escritura periodística.

En efecto -para concentrarnos en lo último-, es un lugar común afirmar que la objetividad resulta ser una condición básica y esperable del periodismo de información. Así lo expresan innumerables - e históricos- manuales de escritura periodística, tanto en español como en otras lenguas (Ross, 1911; Shuman, 1919; McCarthy, 1925; Boyd, 2001; Benavídez y Quintero, 2004; Serra y Ritacco, 2005; Rabatel y ChauvinVileno, 2006; entre muchos otros), y también estudios sobre la historia del periodismo y acerca de las percepciones de las prácticas periodísticas por parte de los mismos profesionales (Schudson,
2001; Hanitzsch, 2007; Mellado y Lagos, 2014; y otros). Más aún, esa propia condición es exigida en el ámbito de las redacciones, con el fin de evitar conflictos judiciales (Tuchman, 1999, p. 202).

Todas estas concepciones son examinadas por autores que estudian el rol del periodista en función de su intervención en cuanto sujeto que elabora informaciones (Himelboim y Limor, 2005; Hanitzsch, 2007; Mellado y Lagos, 2014). En concreto, la dimensión de ese intervencionismo aparece descrita como un continuum que expresa la medida en que el periodista implica sus convicciones y sus juicios de valor al elaborar la información. Uno de los extremos del continuum contempla a quienes se conciben comprometidos con los hechos, con la objetividad y con la imparcialidad. El otro, a quienes se reconocen acreditados para interpretar la realidad que reconstruirán con su discurso. Los preceptos de los especialistas (y de las empresas de medios, que buscan protegerse de eventuales demandas), como se ha señalado, suelen quedar atados al primero de esos extremos.

Con todo, puede suponerse que las prescripciones tradicionales y las ideologías que ellas proyectan no se condicen con las prácticas auténticas de los profesionales a la hora de producir sus textos ${ }^{1}$. Esa es la razón por la cual estudiar los productos escritos del periodismo informativo puede orientar acerca de lo que

1 No queremos decir que los periodistas hagan un uso consciente y deliberado de las marcas que evidencian la intervención de la subjetividad en el texto. En entrevistas informales 
está ocurriendo en este aspecto, más allá de lo que los propios actores crean que debería pasar. Con base en el presupuesto de que los adjetivos subjetivos son la marca más evidente y superficial - es decir, de la superficie del texto- de la intervención de la subjetividad en el discurso (Mellado y Lagos, 2014, p. 2090), consideramos, en esta oportunidad, su ocurrencia en la prensa informativa escrita online de América Latina.

En primer lugar, caracterizamos $\mathrm{y}$ clasificamos los adjetivos calificativos como objetivos, no axiológicos, axiológicos y afectivos (Kerbrat-Orecchioni, 1986), y como descriptivos o evaluativos (Bosque, 1990; Di Tullio, 1997; Giammateo y Albano, 2009). En segundo lugar, estudiamos 490 notas informativas escritas publicadas en diarios online y seleccionadas por el método de semana construida ${ }^{2}: 210$ corresponden a la prensa argentina (clarín.com, lanacion. com.ar y pagina12.com.ar) y 280 pertenecen a cuatro medios diferentes de sendos países latinoamericanos (elcomercio.pe, la-razon.com, latercera.com y elcomercio. com) ${ }^{3}$. Nuestro objetivo consiste en confirmar - o refutar- la hipótesis de que los textos periodísticos informativos no reflejan las prescripciones orientadas a la objetividad, al menos en cuanto a evitar el uso de los adjetivos subjetivos.

\section{Los adjetivos}

El adjetivo es la clase de palabra que modifica al sustantivo o se predica de él. Recibe del propio sustantivo, por concordancia, el género y el número. En el plano sintáctico, el adjetivo admite la posición atributiva, es decir, como complemento del sustantivo dentro del sintagma nominal, tal cual ocurre en (1).

\section{(1) impecable recorrido}

Pero también admite la posición predicativa, o sea, como miembro del predicado, como se ve en (2).

(2) El acto fue considerado como inamistoso.

Existe bastante acuerdo en la clasificación tripartita de los adjetivos (Bosque,

con los propios redactores de algunas de las notas que estudiamos, de hecho, hemos registrado que la mayoría de ellos comulgan explícitamente con la ideología que prescribe la objetividad en el periodismo.

2 La semana construida consiste en elegir, por ejemplo, el lunes de la primera semana del período designado, luego el martes de la segunda semana y así consecutivamente hasta cumplir una semana calendario. Este modo de selección permite obtener una muestra aleatoria de la información y establece un rango de selección relativamente considerable (siete semanas) para la conformación de un corpus de análisis del discurso.

3 Además de los diarios argentinos Clarín, La Nación y Página 12 (seleccionados porque representan tres contratos de lectura complementarios: el contrato con un público general, el contrato con un público conservador, el contrato con un público progresista, respectivamente), estudiamos $L a$ Tercera de Chile, El Comercio del Perú, El Comercio de Ecuador y La Razón de Bolivia en sus versiones online. Estos últimos medios fueron seleccionados por razones de accesibilidad. 
1990; Di Tullio, 1997; Giammateo y Albano, 2009; NGLE, 2009; entre otros): los adjetivos calificativos, los relacionales y los modales y deícticos (también llamados adverbiales). Los últimos dos grupos (relacionales y adverbiales) pueden caracterizarse como "objetivos" desde una perspectiva semántica, y por eso no nos ocuparemos de ellos en este trabajo ${ }^{4}$.

Los adjetivos calificativos suelen ser definidos como aquellos que indican particularidades permanentes o transitorias -ya sea cualidad, ya sea propiedad-de los sujetos denotados por los sustantivos a los que delimitan. Dado que dichas propiedades o cualidades son normalmente incrementales, los adjetivos calificativos admiten ser determinados por un adverbio de grado. Debe notarse, de todos modos, que algunos de ellos (como es el caso de excepcional, por poner un ejemplo) señalan el grado extremo en la escala de la propiedad o la cualidad, por lo que no aceptan la gradualidad.

Siguiendoa Kerbrat-Orecchioni(1986), diremos que los adjetivos calificativos se clasifican, a su vez, en dos grandes grupos, con subdivisiones internas: los objetivos y los subjetivos. Los objetivos son aquellos que clasifican de manera no polémica, según alguna propiedad que se le adjudica al sustantivo como inherente. Tal es el caso de los adjetivos de color, según se observa en (3).

(3) salón amarillo

Los adjetivos subjetivos, por su parte, y siempre siguiendo a Kerbrat-Orecchioni (1986), se dividen en evaluativos (no axiológicos y axiológicos) y afectivos. Los evaluativos no axiológicos, como el de (4), exigen algún tipo de ponderación cualitativa o cuantitativa, sin poner en juego una estimación positiva o negativa.

(4) vínculo fuerte

En efecto, decir de un vínculo que es fuerte, como se plantea en (4), implica desde luego ubicarlo en una escala graduada, pero no dice de él que sea concebido como positivo o negativo gracias a esa calificación.

Desde un punto de vista lógico, puede afirmarse que tanto los objetivos como los evaluativos no axiológicos presentan el grupo nominal formado por sustantivo más adjetivo como que alude a una entidad representada en tanto integrante de la intersección de dos conjuntos. Así, en (3), se une el conjunto de los salones al de las cosas amarillas, y

4 Los adjetivos relacionales vinculan al sustantivo del que se predica con un cierto ámbito, caracterizándolo como miembro de una clase. Normalmente, pueden ser parafraseados por un sintagma preposicional constituido por el sustantivo cuya base léxica se asocia a ese adjetivo (por ejemplo, provincial < de la provincia). Por esta misma condición clasificatoria, no admiten ser determinados por un adverbio de grado $\left({ }^{*}\right.$ bastante provincial). Siempre adoptan la posición posnominal (legislación provincial/*avrovincial legislación). Los adjetivos modales y deícticos, por su parte, cuantifican o ubican deícticamente el sustantivo del que se predican (por ejemplo, mero o próximo). Suelen adoptar la posición prenominal (la mera interrogación, el próximo resultado). 
la entidad individual denotada por el sintagma salón amarillo se ubica en la intersección de esos dos conjuntos. En (4), los conjuntos activados por el sintagma vínculo fuerte son el de los vínculos y el de las cosas fuertes (NGLE, 2009, p. 925) y la entidad individual denotada por el sintagma vínculo fuerte queda localizada en la intersección de ambos.

Es pertinente señalar esta distinción porque, más allá de que los evaluativos no axiológicos aparecen definidos dentro de los adjetivos que esta taxonomía reconoce como subjetivos, tanto los objetivos como los evaluativos no axiológicos se incluyen en la categoría que la NGLE (2009) denomina intersectivos por las razones presentadas en el párrafo anterior (p. 925). Ambos tipos evocan, en definitiva, conjuntos de entidades que se ponen en contacto.

No ocurre lo mismo con los adjetivos evaluativos axiológicos ni con los afectivos. En estos últimos dos casos, no puede hablarse ya de la alusión a la intersección de dos conjuntos de entidades, sino, antes bien, de la caracterización que se hace de la entidad denotada por el sustantivo, pero solo en referencia a ciertos comportamientos o aspectos. $\mathrm{O}$, para decirlo en otros términos, se convoca por medio de ellos una apreciación del hablante que resulta plasmada en esos adjetivos. De esa manera, no se evocan dos conjuntos de entidades para puntualizar la intersección entre ellas, sino, por el contrario, un solo conjunto -el evocado por el sustantivo- del que se expresa una cierta caracterización.
En esa línea, todo adjetivo evaluativo axiológico, como el de (5), manifiesta no solo que el hablante realiza una estimación, sino que esa estimación que se ha realizado encierra un juicio de valor.

(5) paz verdadera

Sin importar si lo que se enuncia coincide con la realidad extralingüística denotada, el adjetivo verdadera que se aplica al sustantivo paz en (5) refleja que el hablante concibe esa paz como algo positivo: verdadera nunca admite una interpretación negativa. $\mathrm{Y}$ es que los adjetivos axiológicos siempre le agregan al sustantivo una calificación que tiene una orientación determinada dentro de un sistema ideológico. Más aún, al hacerlo, también describen al hablante que los emplea; esa es la razón por la cual puede decirse de ellos que son doblemente subjetivos (KerbratOrecchioni, 1986).

Los adjetivos afectivos, finalmente, como el de (6), enuncian una propiedad del objeto que solo puede entenderse en cuanto emergente de una reacción emocional del hablante:

(6) triunfo contundente

Es la visión del hablante la que define que la entidad denotada por el sustantivo admita esta calificación, difícil de definir si se la considera desde una perspectiva lógica o vericondicional. La "contundencia" del triunfo en (6) responde, en concreto, a la impresión que el hablante proyecta sobre su enunciado.

Para decirlo una vez más, frente a los objetivos -que denotan alguna 
propiedad o cualidad como inmanente de aquello que el sustantivo evoca (manzana roja, mesa rectangular) - y los evaluativos no axiológicos (casa grande, café frío), los adjetivos que en este trabajo nombraremos como subjetivos presentan una carga de subjetividad creciente en el orden en que los hemos presentado: si los axiológicos son más subjetivos que los objetivos y los no axiológicos, los afectivos son los más subjetivos de todos.

Pero hay más aún. En la atribución (véase más arriba), esta clasificación se enriquece con la intervención de la sintaxis. $\mathrm{Y}$ es que, si bien los adjetivos calificativos admiten tanto la colocación prenominal como la posnominal, debe señalarse que cada una de esas posiciones provee al adjetivo de sentidos diferentes. La colocación posnominal ofrece un sentido descriptivo. Es decir, cuando el adjetivo está ubicado a la derecha del sustantivo al que modifica, opera "clasificando" o "describiendo" aquello que queda denotado por el sustantivo, tal como ocurre en (7).

(7) funcionario alto

Al igual que en (4), la calificación denota una condición - la de ser alto el funcionario en (7), la de ser fuerte el vínculo en (4) - que está presentada como si todos los sujetos hablantes pudieran acordar acerca de ella. Distinto es el caso de la colocación prenominal, que, como en (8), aporta un sentido de evaluación individual.

\section{(8) alto funcionario}

En otras palabras, cuando se encuentra a la izquierda del sustantivo al que modifica, el adjetivo queda representado como si el hablante se responsabilizara por esa evaluación. Esto significa que el adjetivo en posición prenominal brinda una calificación valorativa (a cargo del hablante) de algún aspecto de la entidad denotada por el sustantivo. En (8), por ejemplo, la altura se refiere a la jerarquía de la función que cumple la entidad calificada y no a la entidad en sí misma (en este caso, el funcionario).

Queda claro así que la posición prenominal se concibe como más subjetiva, en el sentido de que no se presenta a modo de una calificación que debe entenderse como universal - como si fuese aceptada por todos-, sino, por el contrario, como una calificación individual -como si el enunciador exhibiese explícitamente su aporte personal en esa calificación-. Un ejemplo que clarifica esta diferencia es la oposición entre (9) y (10).

(9) militante viejo

(10) viejo militante

En (9), el adjetivo se relaciona estrictamente con la edad. En (10), el adjetivo se asocia con alguna característica menos ponderable, como el compromiso con la militancia o la relevancia de la militancia; no se vería una contradicción, incluso, al afirmar que ese militante joven es un viejo militante.

Este segundo enfoque, desde luego, complejiza el esquema de adjetivos que tomamos de Kerbrat-Orecchioni (1986). Todo adjetivo que, clasificado aisladamente como objetivo, se ubique en posición prenominal subirá un grado en la escala de subjetividad y será 
catalogado como evaluativo no axiológico. Todo adjetivo que, clasificado aisladamente como evaluativo no axiológico, se ubique en posición prenominal subirá un grado en la escala de subjetividad y será catalogado como axiológico. Todo adjetivo que, clasificado aisladamente como evaluativo axiológico, se ubique en posición prenominal subirá un grado en la escala de subjetividad y será catalogado como afectivo (véase la tabla 1).

En el parágrafo siguiente, aplicaremos esta clasificación a los textos reales de nuestro corpus.

Tabla 1. Clasificación de los adjetivos

\begin{tabular}{|c|c|c|c|}
\hline \multicolumn{4}{|c|}{ Clasificación de los calificativos a partir de Kerbrat-Orecchioni (1986) y de la NGLE (2009) } \\
\hline \multirow[t]{3}{*}{ Objetivos } & \multicolumn{3}{|l|}{ Subjetivemas } \\
\hline & \multicolumn{2}{|l|}{ Evaluativos } & \multirow[t]{2}{*}{ Afectivos } \\
\hline & No axiológicos & Axiológicos & \\
\hline tío soltero & edificio alto & alto conocimiento & escena horripilante \\
\hline manzana verde & profesora joven & amistad leal & incendio pavoroso \\
\hline calesita redonda & casa grande & rostro bello & bello espectáculo \\
\hline \multicolumn{2}{|c|}{ Intersectivos } & \multicolumn{2}{|c|}{ No intersectivos } \\
\hline \multicolumn{2}{|c|}{ - subjetivo } & \multicolumn{2}{|c|}{+ subjetivo } \\
\hline
\end{tabular}

Elaboración propia

\section{Análisis del corpus: el uso de adjetivos subjetivos en la prensa informativa}

Nuestro corpus totaliza casi 240000 palabras. Para conformarlo, seleccionamos 70 notas de cada uno de los siete medios estudiados aplicando el método de semana construida (10 notas por día de la semana), que fueron tomadas de cada homepage, en algún momento del día: contando de izquierda a derecha y de arriba hacia abajo, se trataba de las primeras diez notas informativas. Una vez constituido el corpus, elaboramos una grilla de codificación en la que computamos la cantidad total de palabras de cada nota y registramos los adjetivos en cada una de ellas.

Dado que nuestra hipótesis se enfoca en la intervención de la subjetividad, tras haber realizado una codificación rigurosa de todos los adjetivos de la muestra - controlada por un codificador alternativo-, tomamos exclusivamente los adjetivos no intersectivos - esto es, los axiológicos y los afectivos, que, como queda dicho, son los que denominamos subjetivos en este trabajo- para reconocer la magnitud de su presencia en la prensa informativa escrita digital y para establecer luego comparaciones entre los 
medios que analizamos. Esa ocurrencia fue registrada porcentualmente: si, por ejemplo, consignamos 5 adjetivos subjetivos no intersectivos en una nota de 500 palabras, el porcentaje que calculamos para esa nota fue del $1 \%$. Una vez analizadas las 70 notas correspondientes al subcorpus de cada medio, obtuvimos el promedio de porcentaje de ocurrencia de estos adjetivos en él.

Para especificar mejor el procedimiento, es necesario agregar que, en ningún caso, consideramos los adjetivos incluidos en citas directas (entrecomilladas). Desde luego, es cierto que el propio locutor es quien selecciona la cita que va a introducir en su texto ${ }^{5}$, pero no podría atribuirse a la intervención de su subjetividad el empleo de un adjetivo por parte de una voz que le corresponde a un sujeto distinto de él.

En cuanto a la cantidad de palabras que se tuvieron en cuenta en cada caso, siempre se trató del total efectivo, incluidas las citas directas (entrecomilladas). Esta decisión tiene que ver con que algunas pocas notas del corpus estaban plagadas de citas directas, y extraerlas para hacer el cálculo correspondiente habría significado un desbalance sustantivo en este punto en relación con la media. El hecho de generalizar esta norma, de todos modos, pretende aminorar cualquier margen de error significativo al respecto.

En la tabla 2, ubicamos cada uno de los medios estudiados con el porcentaje promedio correspondiente de adjetivos

Tabla 2. Cálculo de ocurrencia de adjetivos subjetivos en medios de América Latina

\begin{tabular}{lllcc}
\hline \multicolumn{1}{c}{ País } & \multicolumn{1}{c}{ Medio } & $\begin{array}{c}\text { Promedio de } \\
\text { cantidad de } \\
\text { palabras por nota }\end{array}$ & $\begin{array}{c}\text { Promedio de porcentaje } \\
\text { de ocurrencia de } \\
\text { adjetivos subjetivos }\end{array}$ \\
\hline 1 & Chile & La Tercera (latercera.com) & 467 & 1,63 \\
2 & Argentina & Clarín (clarín.com) & 583 & 1,05 \\
3 & Argentina & Página 12 (pagina12.com.ar) & 671 & 0,85 \\
4 & Ecuador & El Comercio (elcomercio.com) & 502 & 0,66 \\
5 & Perú & El Comercio (elcomercio.pe) & 377 & 0,60 \\
6 & Bolivia & La Razón (la-razon.com) & 400 & 0,60 \\
7 & Argentina & La Nación (lanacion.com.ar) & 565 & 0,43 \\
\hline
\end{tabular}

Elaboración propia

5 Por medio de las declaraciones de las fuentes, es claro que el periodista puede conseguir que se plasme lo que él mismo piensa (Tuchman, 1999, p. 206). 
subjetivos no intersectivos por total de palabras de la nota.

Como se ve en la tabla 2, en la progresión que va de mayor a menor empleo de adjetivos subjetivos, La Tercera de Chile se ubica en primer lugar, seguida de dos medios de Argentina: Clarín y Página 12. A continuación, se encuentra un medio de Ecuador, El Comercio, seguido de $E l$ Comercio de Perú y La Razón de Bolivia. En la última posición está La Nación de Argentina.

Lo primero que se puede constatar aquí es que estos hallazgos no concuerdan totalmente con los del equipo coordinado por la chilena Claudia Mellado (Mellado et al., 2016) ${ }^{6}$. En efecto, estos autores afirman:

La cuantificación de indicadores permite analizar la presencia de la voz pe- riodística en las historias analizadas, como surge de los datos descriptivos de la tabla 5.2 [figura 1]. Los resultados muestran que, en términos globales, el modelo intervencionista está más presente en Argentina y Chile que en Brasil, Ecuador y México. Cuando se analizan los indicadores por separado, el uso de adjetivos, la interpretación y la opinión son los que más aparecen en todos los países. En el caso argentino, en más del $20 \%$ de las noticias analizadas, el periodista presenta una opinión sobre el tema de la historia expresando un juicio de valor al respecto. El uso de adjetivos calificativos es particularmente alto en Argentina y en Chile, con presencia en dos tercios de las noticias. (p. 67)

Nuestros resultados -admitiendo que no siempre tomamos los mismos medios - se contradicen parcialmente con los suyos. En primer lugar, no

Figura 1. Captura de los hallazgos de Mellado et al. (2016)

Tabla 5.2. Modelo intervencionista en la prensa latinoamericana

\begin{tabular}{|l|c|c|c|c|c|}
\hline \multicolumn{1}{c|}{} & Argentina & Brasil & Chile & Ecuador & México \\
\hline Media / SD & $0,29 / 0,206$ & $0,12 / 0,182$ & $0,23 / 0,194$ & $0,12 / 0,161$ & $0,14 / 0,214$ \\
\hline Opinión del periodista & $20,2 \%$ & $8,8 \%$ & $14,5 \%$ & $12,5 \%$ & $15,9 \%$ \\
\hline Interpretación & $44,6 \%$ & $30,2 \%$ & $24,8 \%$ & $42,2 \%$ & $13,7 \%$ \\
\hline Propuesta/Demanda & $1,9 \%$ & $4,0 \%$ & $6,2 \%$ & $1,9 \%$ & $4,6 \%$ \\
\hline Adjetivos & $71,8 \%$ & $16,9 \%$ & $67,3 \%$ & $4,7 \%$ & $33,9 \%$ \\
\hline Primera persona & $1,7 \%$ & $1,3 \%$ & $2,4 \%$ & $1,0 \%$ & $1,4 \%$ \\
\hline
\end{tabular}

Fuente: Mellado et al. (2016)

6 En buena medida, los resultados de Mellado et al. (2016) constituyeron el disparador para llevar adelante nuestra investigación. Debe señalarse, de todos modos, que ese trabajo abarca una serie de índices, en tanto que el nuestro se ocupa en esta oportunidad de uno solo. 
podemos afirmar que todos los medios en Argentina se comporten de manera similar. Es más, hay diferencias sustantivas en el uso de los adjetivos subjetivos no intersectivos entre Clarín y Página 12, que están entre los que más usan esta clase de adjetivos, por un lado, con $\mathrm{La}$ Nación, que es el medio que menos emplea estos adjetivos, por el otro. En segundo lugar, nuestro análisis del medio ecuatoriano no coincide con la estimación que realiza el grupo de la académica chilena. La tabla 3 muestra el cotejo entre los resultados comparables (véase Mellado et al., 2016, p. 67, y la tabla 2) entre ambos estudios (para el caso argentino, nosotros hacemos un promedio).

Tabla 3. Resultados comparados con el trabajo de Mellado et al. (2016)

\begin{tabular}{lcc}
\hline & Mellado et al. (2016) & Nuestros resultados \\
\hline Argentina & 71,8 & 0,77 \\
Chile & 67,3 & 1,63 \\
Ecuador & 4,7 & 0,66 \\
\hline
\end{tabular}

Fuente: Comparación de los datos consignados en la tabla 2 y los del trabajo de Mellado et al. (2016)

Figura 2. Resultados de Mellado et al. (2016)

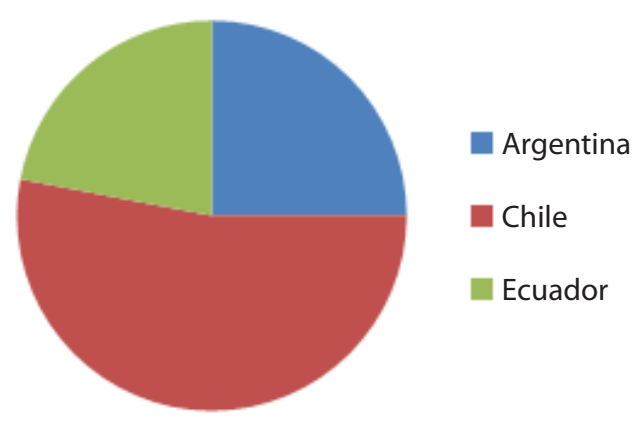

Fuente: Mellado et al. (2016) 
Figura 3. Nuestros resultados

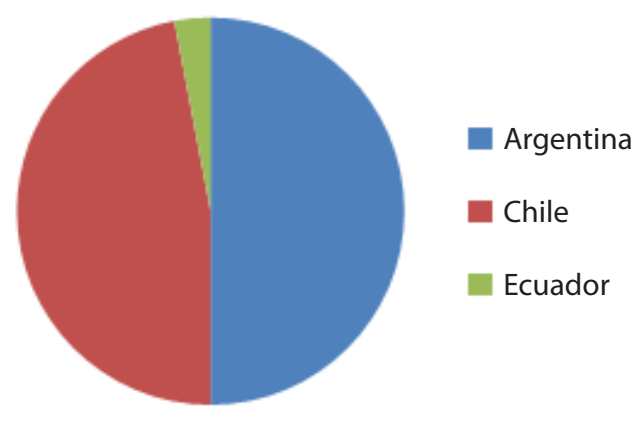

Elaboración propia

Tabla 4. Resultados comparados con el trabajo de Mellado et al. (2016), normalizados

\begin{tabular}{lcc}
\hline & Mellado et al. (2016) & Nuestros resultados \\
\hline Argentina & $50 \%$ & $25 \%$ \\
Chile & $47 \%$ & $53 \%$ \\
Ecuador & $3 \%$ & $22 \%$ \\
\hline
\end{tabular}

Elaboración propia

Para que se vea más claramente el contraste, los normalizamos ${ }^{7}$ entre sí y los comparamos con dos gráficos (figuras 2 y 3). $\mathrm{Y}$ esto mismo es lo que representamos en la tabla 4.

Desde luego, es importante señalar que los autores con cuyo trabajo estamos contrastando el nuestro simplemente consideran el porcentaje de notas de su corpus con presencia de adjetivos calificativos: si de 100 notas se hallan adjetivos en 40 , se registra un $40 \%$ en la tabla correspondiente de Mellado et al. (2016, p. 67). Por nuestra parte, no solo hicimos una clasificación pormenorizada del tipo de adjetivos para computar exclusivamente los subjetivos no intersectivos, sino que también - como ya se

7 La normalización consiste en tomar como totalidad la suma de los tres resultados y luego dividir el círculo en porciones según el porcentaje de ese total que corresponde a cada uno. Esto permite reconocer la magnitud del contraste entre los empleos de adjetivos por parte de los medios seleccionados de estos países. 
ha dicho-, numeramos y consignamos cada uno de estos adjetivos presentes en cada nota, para luego anotar porcentualmente la cantidad de adjetivos sobre el total de palabras. De allí la notoria diferencia numérica de los porcentajes.

La perspectiva que guía nuestra tarea es que, más que la clase general de los calificativos, es el tipo específico de adjetivos presentes lo que revela la intervención de la subjetividad en los discursos. Para decirlo de otro modo, no es la presencia cruda de adjetivos calificativos - como en la iglesia redonda o el vínculo fuerte- lo que pone de manifiesto la subjetividad, sino, antes bien, el empleo de adjetivos con carga subjetiva más ideológica, como es el caso de naturaleza dudosa o panorama desolador.

A este análisis más cuantitativo debe agregarse uno más cualitativo. De hecho, en algunos casos, cantidad e intensidad de la carga subjetiva parecen correlacionarse: los medios de nuestro corpus que proyectan más fuertemente la subjetividad desde el punto de vista del contenido léxico - siempre tomando el caso de los adjetivos- son Página 12 de Argentina y La Tercera de Chile. También Clarín, aunque en menor medida.

(11) El perfil de los oradores marca claramente la politización: el fiscal Germán Moldes, un rabioso ${ }^{8}$ adversario del kirchnerismo; el escritor Federico Andahazi, de los pocos intelectuales alineados con el PRO, y Luis Czyzewski, papá de Paola, muerta en el atentado contra la AMIA. (Página 12, 18 de enero del 2017)

(12) La neolengua, como forma de controlar el pensamiento, está más viva que nunca, esterilizando el mundo de las ideas con odios infames y muros abyectos. (Página 12, 26 de enero del 2017)

(13) Allí, el áspero mediocampista de Boca, Pablo Pérez, le tiró una patada al mexicano Hernández. (Clarín, 3 de febrero del 2017)

(14) También dejó novelas y poesías deliciosas, impregnadas de la más dura crítica de la época, pero también de confianza en la especie humana. (Página 12, 2 de enero del 2017)

(15) Sin embargo, según la acusación, el verdadero propietario sería Lula, que lo habría recibido a cambio de "favores" hechos desde el poder a esa constructora, una de las implicadas en la monumental red de corrupción de Petrobras que investiga el juez Moro. (La Tercera, 10 de mayo del 2017)

Calificar a un adversario de rabioso o reputar a ciertos odios de infames y a ciertos muros de abyectos, como ocurre en (11) y (12), y usar una sinestesia para adjetivar a un jugador de fútbol, como en (13), o para describir la literatura, como en (14), no solo patentiza la condición fuertemente pasional de un texto, sino que también revela en él una vocación literaria. Y caracterizar como monumental una red de corrupción, tal cual ocurre

8 En todos los casos, destacamos en negrita el adjetivo del que hablaremos a continuación. 
en (15), deja en claro que el texto se presenta como manifiestamente personal (esto último acentuado por la posición prenominal que adopta el adjetivo).

De cualquier manera, no son estos los únicos tipos de uso de los adjetivos subjetivos no intersectivos de este corpus. En algunos casos, de hecho, hay recurrencia de clichés.

(16) La nueva política de Trump provocó airadas protestas en Nueva York, donde anoche mil personas se manifestaron en contra de su política. (Clarín, 26 de enero del 2017)

(17) Estremecedor accidente en una práctica de bungee jumping se viraliza en las redes sociales. (La Razón, 24 de abril del 2017)

(18) González llegó a presidir el club deportivo de pasado glorioso. ( $\mathrm{La}$ Tercera, 25 de mayo del 2017)

(19) La carrera Últimas Noticias $15 \mathrm{~K}$, que se realizó este domingo 4 de junio del 2017, tuvo un duelo emocionante entre los atletas Daniel Muindi de Kenia y Cristian Pacheco del Perú. (El Comercio, 4 de junio del 2017)

En efecto, airadas protestas, estremecedor accidente, pasado glorioso o duelo emocionante en (16) hasta (19) no son más que lugares comunes. Usualmente listadas y estigmatizadas por los especialistas, estas frases repetidas suelen ser empleadas como comodines en la escritura periodística, aun cuando, por trilladas, ya se encuentren vacías de contenido.

En otros casos, en fin, el adjetivo subjetivo se utiliza en función de una exposición enfática.
(20) La candidata es una ferviente militante de los valores religiosos, muy conservadora y defensora de la educación privada. (La Nación, 3 de febrero del 2017)

(21) Ecuador le pone fin al inusual ciclo político de Correa (La Nación, 19 de febrero del 2017)

(22) En Caracas, el "plantón", que los dirigentes opositores aclararon que no será un bloqueo de vías con barricadas, sino una concentración de personas, se realizará en una estratégica autopista de la ciudad, en el este, y en las principales vías de cada estado. (El Comercio, 24 de abril del 2017)

(23) Detrás está Guabirá, que suma 20, luego de lograr un heroico empate 3-3. (La Razón, 24 de abril del 2017)

Como se puede constatar de (20) a (23), la ocurrencia del adjetivo en posición prenominal o evaluativa parece convocada a enfatizar lo que se habría expresado con el mismo adjetivo pospuesto. Así, una ferviente militante parece más ferviente que una militante ferviente, un inusual ciclo parece más inusual que un ciclo inusual, una estratégica autopista es más estratégica que una autopista estratégica y un heroico empate es más heroico que un empate heroico.

En definitiva, lo que queda en evidencia es que, lejos de estar ausente en el discurso periodístico en función de las recomendaciones de la literatura prescriptiva y de los editores en cuanto garantes empresariales de la práctica profesional, los adjetivos no intersectivos que hemos llamado aquí subjetivos ocurren y enriquecen - la mayoría de las veces- el texto de la información. 
En algún sentido, la ocurrencia de este tipo de adjetivos - seguramente en concurso con otras marcas- colabora con la construcción de un texto que palpita y que trasunta humanidad. No se trata del texto aséptico que pudieran producir los robots, sino, más bien, de un texto que deja traslucir en su génesis una presencia viva, que razona, pero que también siente.

No solo eso. La ocurrencia de estos adjetivos ofrece al lector una credencial patente de la orientación del texto: este revela así su postura ideológica de manera transparente. Ningún texto habla de un rabioso adversario si su contenido va a representar a ese adversario positivamente. Ni hablará de un heroico empate si su contenido hace referencia a un partido anodino. En tiempos de cuestionamiento de la credibilidad de los medios, un recurso como este puede ser interpretado en línea con la búsqueda de recomposición del contrato de confianza entre los lectores o usuarios $^{9}$ y el texto periodístico: los adjetivos actúan como una advertencia que debe ser leída; el texto, también por medio de ellos, expone su orientación.

\section{Conclusión}

Beneficiaria del cogito cartesiano, la noción de sujeto constituye un tema de interés actual para diversos campos disciplinares, desde la lingüística hasta la sociología. El sujeto racional al que alude esa noción se instaura como la figura capaz de reproducir en su discurso, a modo de un espejo, la realidad tal y como es, sin distorsiones ni tergiversaciones, por medio de enunciados que la describen fielmente. Se entiende así que es objetivo lo que pertenece al objeto del pensamiento y subjetivo lo que se asocia con el sujeto pensante. En esa misma línea, la mayoría de las prescripciones relativas a la escritura periodística suelen promover la producción de discursos objetivos y objetar la intervención de la subjetividad. Sin embargo, nuestra hipótesis es que esa concepción queda en entredicho cuando se analizan las producciones concretas de los periodistas.

Para confirmar esta hipótesis, en este trabajo hemos caracterizado y catalogado los adjetivos calificativos subjetivos como no axiológicos, axiológicos y afectivos (Kerbrat-Orecchioni, 1986), y como descriptivos o evaluativos (Bosque, 1990; Di Tullio, 1997; Giammateo y Albano, 2009), para luego reconocerlos y contabilizarlos en 490 notas informativas escritas aparecidas en diarios online de Argentina, Chile, Ecuador, Perú y Bolivia.

Si se admite que el empleo de adjetivos constituye una muestra de la presencia de la subjetividad en el texto, la ocurrencia de adjetivos con una alta carga de subjetividad -los axiológicos y

9 Llamamos usuarios a los lectores en pantalla. 
los afectivos (Kerbrat-Orecchioni, 1986), que hemos categorizado como subjetivos no intersectivos (NGLE, 2009) - en la prensa escrita online pone en evidencia que el rol del periodismo informativo en América Latina no asume la objetividad en los términos definidos por las prescripciones tradicionales de este campo disciplinario.

Nuestros resultados muestran, en definitiva, que los productos concretos de la tarea periodística online se distancian en este aspecto de las prescripciones habituales. Y la interpretación que respaldamos se asocia con una visión del periodismo como orientado a cumplir con una misión social, e investido de un compromiso individual con su audiencia. Una visión, en suma, concordante con un concepto de sujeto ya no definitivamente racional, sino más bien relacional y complejo.

\section{Referencias}

Amado, A., Arroyave, J., Benítez J. L., Chavero, P., Garcés, M., Hughes, S., Márquez, M., Mellado, C., Moreira, S. V., y Oller, M. (2016). Periodismos latinoamericanos: perfil y roles profesionales. En A. Amado (Ed.), El periodismo por los periodistas (pp. 11-25). Montevideo: Konrad Adenauer Stiftung; Infociudadana.

Benavídez, J. L., y Quintero, C. (2004). Escribir en prensa. Madrid: Pearson.

Bosque, I. (1990). Las categorías gramaticales. Madrid: Síntesis.

Boyd, A. (2001). Broadcast Journalism. Oxford: Focal Press.

Di Tullio, Á. (1997). Manual de gramática del español. Buenos Aires: Edicial.

Giammateo, M., y Albano, H. (2009). Cómo se clasifican las palabras. Buenos Aires: Littera.

Hanitzsch, T. (2007). Deconstructing Journalism Culture: Toward a Universal Theory. Communication Theory, 17, 267-385. https://doi.org/10.1111/j.14682885.2007.00303.x

Himelboim, I., y Limor, Y. (2005). The Journalistic Societal Role: An International Comparative Study of 242 Codes of Ethics. En 55 th Annual Conference of the International Communication Association. Nueva York, NY.

Hyland, K. (2002). Authority and Invisibility: Authorial Identity in Academic Writing. Journal of Pragmatics, 8(34), 1091-1112.

Kerbrat-Orecchioni, C. (1986). La enunciación. De la subjetividad en el lenguaje. Buenos Aires: Hachette.

McCarthy, J. (1925). The Newspaper Worker. Nueva York, NY: Frank-Maurice Inc.

Mellado, C., y Lagos, C. (2014). Professional Roles in News Content: Analyzing Journalistic Performance in the Chilean National Press. International Journal of Communication, 8, 2090-2112. 
Mellado, C., Márquez-Ramírez, M., Oller Alonso, M., Mick, J., y Amado, A. (2016). Puesta en práctica de los roles periodísticos: un estudio comparado de Argentina, Brasil, Chile, Ecuador y México. En A. Amado (Ed.), El periodismo por los periodistas (pp. 64-71). Montevideo: Konrad Adenauer Stiftung; Infociudadana.

Rabatel, A., y Chauvin-Vileno, A. (2006). La question de la responsabilité dans l'écriture de presse. Semen, 22. Recuperado de http://semen.revues.org/2792

Real Academia Española; Asociación de Academias de la Lengua Española. (2009). Nueva gramática de la lengua española. Madrid: Espasa Libros.

Ross, C. (1911). The Writing of the News. Nueva York, NY: Holt \& Co.

Schudson, M. (2001). The Objectivity Norm in American Journalism. New Media \& Society, 2(2), 149-170.

Serra, A., y Ritacco, E. (2005). Curso de periodismo escrito. Buenos Aires: Atlántida.

Shuman, E. (1919). Practical Journalism. Nueva York, NY; Londres: Appleton \& Co.

Tuchman, G. (1999). La objetividad como ritual estratégico: un análisis de las nociones de objetividad de los periodistas. CIC, 4, 199-217. 
